Careless Response Processes are Heterogeneous: Comment on Goldammer et al. (2020)

\author{
Alexander J. Denison and Brenton M. Wiernik \\ Department of Psychology, University of South Florida
}

\begin{abstract}
Author Note
Alexander J. Denison (iD https://orcid.org/0000-0002-4291-8325

Brenton M. Wiernik (iD https://orcid.org/0000-0001-9560-6336

Correspondence concerning this article should be addressed to Alexander J. Denison or Brenton M. Wiernik, Department of Psychology, University of South Florida, 4202 East Fowler Ave, Tampa, FL 33620. Email: adenison@usf.edu or brenton@wiernik.org
\end{abstract}

Submitted to Meta-Psychology. Participate in open peer review by commenting through hypothes.is directly on this preprint. The full editorial process of all articles under review at MetaPsychology can be found following this link:

https://tinyurl.com/mp-submissions

You will find this preprint by searching for the first authors name. 


\begin{abstract}
Goldammer et al. (2020) examined the performance of careless response detection indices by experimentally manipulating survey instructions to induce careless responding, then compared the ability of various indices to detect these induced careless responses. Based on these analyses, Goldammer et al. concluded that metrics designed to detect overly consistent response patters (i.e. longstring and IRV) were ineffective. In this comment, we critique this conclusion by highlighting critical problems with the experimental manipulation used. Specifically, Goldammer et al.'s manipulations only encouraged overly inconsistent, or random, responding and thus did not induce the full range of careless response behavior that is present in natural careless responding. As such, it is unsurprising that metrics designed to detect overly consistent responding did not appear to be effective. Because the full range of careless behavior was not induced, Goldammer et al.'s study cannot address the utility of longstring or similar metrics. We offer recommendations for alternative experimental manipulations that may produce more naturalistic and diverse careless responding.
\end{abstract} Keywords: careless responding, insufficient effort responding, data cleaning, methodology 


\section{Careless Response Processes are Heterogeneous: Comment on Goldammer et al. (2020)}

Goldammer et al. (2020) presented two studies aimed at investigating the performance of various methods for detecting careless respondents. Their first study experimentally manipulated response patterns using various instruction sets and examined the performance of commonly used careless response detection methods. Based on these results, Goldammer et al. provided recommendations about which detection methods are effective and ineffective. In this comment, we discuss several important limitations of the careless response manipulations used in this study, whether these manipulations produced behavior consistent with natural careless responding, and the appropriateness of Goldammer et al.’s recommendations.

\section{Defining Careless Responding}

Careless or insufficient effort responding ${ }^{1}$ is part of a larger construct of carelessness or inattentiveness, which has been used to describe response behaviors that arises when individuals are not motivated to give honest, thoughtful responses to questions (Johnson, 2005). Careless responding occurs when this carelessness results in individuals answering items in a content non-responsive manner - i.e. without paying attention to the content or instructions of the items (Curran, 2016; Meade \& Craig, 2012; Nichols et al., 1989). This is distinct from other aberrant response patterns such as faking, which is content-responsive. When faking, individuals provide invalid data, but their responses are contingent on the content of the items they are responding to (Nichols et al., 1989).

Content non-responsive behavior has a long history of study in psychology, dating back over 30 years to examinations of the detecting such behavior in the MMPI (Baer et al., 1997; Berry et al., 1992; Nichols et al., 1989) and examining factors arising from negatively keyed items (Schmitt \& Stuits, 1985). The construct of careless responding grew out of these early investigations as a way to

\footnotetext{
${ }^{1}$ The terms careless responding and insufficient effort responding are used to refer to the same underlying construct in almost all cases. In this paper we opt to use the term careless responding when discussing the construct.
} 
describe content non-responsive behavior that was due to a lack of motivation. It is now well accepted that this behavior can take on two different forms - overly consistent or overly inconsistent responding. Overly inconsistent responding can be conceptualized as randomness or random responding, as it is assumed that individuals select their response to each item completely at random. Conversely, overly consistent responses follow some pattern, such as responding with the same anchor point to every item or varying responses in some pattern, such as " $1,2,1,2,1,2 \ldots$ ". or " 1,2 , 3, 4, 5, 4, 3, 2, 1..." (Curran, 2016; Meade \& Craig, 2012).

While careless responding is the most commonly investigated construct when examining content-nonresponsive behavior, it is important to note that not all studies in the literature investigate this construct in its entirety. Specifically, there are many studies that explicitly investigate random responding (e.g. Berry et al., 1992; Credé, 2010; Osborne \& Blanchard, 2011) with no investigation of consistent responding. Although random responding is one piece of the careless responding construct, it is not equivalent to the construct of careless responding as a whole, and any investigation of purely random responding will overlook a large chunk of the careless responding construct. The conflation of random responding with all careless responding is something most authors are careful to avoid. Indeed, in all of the aforementioned examinations of random responding the authors are careful to separate random and careless responding, and at most talk about carelessness as the underlying cause of random responding.

These two conceptualizations of careless response behavior are reflected in the metrics that have been developed to catch these respondents. As Goldammer et al. note there are two general categories of indirect careless response metrics. The first category is invariability metrics, which detect overly consistent response behavior. This category includes longstring analysis and intraindividual variability, among other techniques. The second category is consistency metrics, 
which detect overly inconsistent behavior. This category includes a person's response reliability and semantic or psychometric antonyms/synonyms. See Goldammer et al. (2020) for a full description of these metrics.

\section{Inducing Careless Responding}

Given the above definition of careless responding as content non-responsive, it is critical to ensure that any manipulation intended to induce careless behavior is consistent with this definition. It is also important to consider whether experimentally induced behavior provides a good proxy for what that behavior is like in the real world. For comparison, research on the aberrant response pattern of faking has consistently found that faking induced through researcher instructions does not approximate non-directed or "real-world" faking (Kuncel et al., 2011; Viswesvaran \& Ones, 1999). It is questionable whether either of Goldammer et al.'s manipulations induce behavior that is a good proxy for real-world careless responding or that is even consistent with the definition of careless responding.

Goldammer et al.'s first manipulation is “opposite responding," wherein participants are instructed to respond to items using the opposite scale point they would normally choose. This response pattern clearly violates the definition of careless responding as it is not content nonresponsive behavior; participants are instructed to attend to the item content and then to respond in a

specific aberrant way based on what they would normally answer. That is, respondents must attend to the content of a given item to generate their initial response, then reverse that response. Although these data may be considered invalid, as half of their responses will not align with the other half, they are produced from content-responsive, not careless, behavior.

This is not to say that careless response indices will be unable to detect such behavior; in fact, we can see that the metrics designed to detect inconsistent response patterns do a good job of capturing this "opposite responding" pattern. However, this result is unsurprising because this 
manipulation, by design, makes a participant's responses inconsistent. Conversely, it would be nearly impossible for the "opposite responding" manipulation to produce an overly consistent response pattern that would be detected by longstring analysis, for example. In fact, longstring would only detect the opposite responding pattern if a participant happened to respond to all items at the midpoint. Consistency indices were not designed to detect an "opposite response" pattern, and it is neither unexpected nor informative that they, in fact, do not detect such behavior. Thus, the results from this manipulation do not have any bearing on the power of inconsistency or consistency indices to detect careless responding because the responses are not careless. ${ }^{2}$

Goldammer et al.'s second manipulation is “random responding." Participants in this condition were instructed on alternating pages to either "complete the questions below exactly as they apply to you" or to "choose any response option, no matter whether it applies to you or not" (p. 4). It is again unclear what real-world response pattern this represents. Under what conditions would participants repeatedly start and stop attending to item content? Even if participants were partially careless, it is unlikely they would exhibit this in alternating blocks of responses. By repeatedly changing the instructions from careful to random and vice versa, this manipulation will increase the within-person variability of responses, but it is not clear that these responses will resemble real-world random responding, much less careless responding as a whole.

Even if these instructions are assumed to reasonably approximate random responding, these responses would nevertheless not reflect the full scope of response behavior exhibited in real-world careless responding. Previous work has extensively documented that careless responding is not

\footnotetext{
${ }^{2}$ It is somewhat difficult to determine what sort of real-world response pattern the "opposite responding" is intended to reflect. Rarely would a person be expected to actively switch their scale point use partway through a survey. One possibility would be if the anchor points for scales were reversed midway through a multi-part survey and the participant did not notice this reversal. However, such a change would go against general good survey design practices (Stern et al., 2007) and would likely be better simulated by instructing respondents to respond normally and randomly reverse-coding items for a subset of respondents.
} 
simply responding randomly to items, but can also manifest as certain forms of patterned or consistent responding (Curran, 2016; Johnson, 2005; Meade \& Craig, 2012). For example, a respondent might try to complete a survey quickly by clicking the same scale item repeatedly. Invariability metrics are designed specifically to capture this type of behavior because we know overly consistent responding is a type of behavior careless respondents engage in. Because the manipulation used by the authors is unlikely to produce overly consistent behavior, it is again unsurprising that the invariability metrics failed to detect these respondents. ${ }^{3}$ As such, the data have no bearing on the utility of invariability indices because invariability indices were not designed to detect the random or inconsistent behavior induced by the authors manipulation.

\section{Are Invariability Metrics Useless?}

A key takeaway from our examination of both of Goldammer manipulations is that the response patterns they produce will be highly inconsistent. Thus, it is unsurprising that Goldammer et al. conclude that metrics designed to detect overly inconsistent behavior (consistency metrics) are effective at detecting careless responding, whereas the invariability metrics designed to detect overly consistent responding are not. This finding does not mean that invariability metrics are useless, but rather that the manipulations were not designed to produce consistent responding.

Goldammer et al.'s conclusions in their Study 1 and subsequent recommendations against use of invariability indices are especially problematic because we know that actual careless respondents $d o$ sometimes produce overly consistent response patterns. For example, Johnson (2005) identified that $3.5 \%$ of participants in their dataset responded by selecting the same response option repeatedly throughout the survey; similar patterns have been noted in other studies of careless

\footnotetext{
${ }^{3}$ The actual participation instructions used did not explicitly state that participants should respond at random, but it seems highly likely that participants would interpret these instructions this way. Indeed, Golammer et al. appeared to assume as much, as their descriptions of the manipulation clearly communicate that they thought participants were engaging in random responding.
} 
responding (Curran, 2016; Meade \& Craig, 2012). Therefore, applying only consistency metrics in a real dataset (as done in Goldammer et al.'s Study 2) will overlook a potentially large portion of careless respondents who are engaging in overly consistent response behavior.

\section{How Can We Experimentally Study Careless Responding?}

Above, we critique Goldammer et al.'s manipulations as unlikely to produce response behavior that resembles real careless responding. This raises the question of how careless responding could be experimentally studied. We offer several possibilities.

First, rather than instructing participants to respond in a specific way, researchers can instead instruct participants to optimize their responses based on a specific goal that is relevant for the population being studied. For example, study participants recruited for extra credit in university classes or platforms like MTurk might aim to complete surveys as quickly as possible to receive their compensation. To simulate such a strategy, participants could be instructed to "answer items as quickly as possible" or to "answer items as quickly as possible, while still appearing to respond carefully." (cf. Huang et al., 2012). This type of instruction may more accurately reflect the types of thought processes that lead to real-world careless responding, and participants might engage in a variety of behaviors to achieve this goal (e.g., random responding, patterned responding).

This approach is not foolproof. It may be difficult to write instructions that capture the myriad of factors that research participants simultaneously weigh when choosing a response strategy (cf. directed "fake good" instructions do not produce the same response strategies test takers use in real high-stakes settings; Kuncel et al., 2011; Viswesvaran \& Ones, 1999). Thus, a second approach might be to use manipulations designed to decrease the probability that individuals respond carelessly. For example, participants in one condition could be warned that "The researchers will be able to detect if you have responded carelessly. You will not receive compensation if you respond carelessly.” (cf. Gibson, 2019; Huang et al., 2012). Researchers could also employ a virtual presence 
(such as a human, or more abstract entity) to make participants feel monitored, in addition to warning them (cf. Ward \& Pond, 2015). When participants are made to feel monitored or warned, carelessness rates should be lower. Accordingly, careless responding indices would be expected to detect lower rates of carelessness in these conditions, compared to control conditions without a warning. Similarly, performance of careless responding indices could be compared across data collected in comparatively high-stakes (e.g., a job application) versus low-stakes (e.g., an extra credit or MTurk study) settings; careless responding rates would be expected to be lower in high-stakes contexts.

A third approach might be to directly ask participants whether they responded to a survey carelessly and then examine whether careless responding indices can detect participants who responded affirmatively to this item (cf. a single item asking participants about their data quality can effectively detect a high percentage of careless respondents; Meade \& Craig, 2012). Last, a fourth approach might be to induce content non-responsive responses by using items with nonsensical or blank content (cf. Maul, 2017; but see also Rhemtulla et al., 2017 and Curran \& Hauser, 2019).

Each of these approaches has potential advantages and disadvantages, and experimental studies of careless responding should triangulate results across multiple approaches. However, any study of careless responding must ensure that the responses accurately represent the real psychological decision-making and response processes used by real careless responders. In the interim, applied researchers are advised to consider a variety of careless responding metrics, including both consistency metrics and invariability metrics, to identify the full range of potentially invalid responses. 


\section{Disclosures}

Conflict of Interest and Funding: None

\section{Author Contributions}

Conceptualization: Alexander J. Denison and Brenton M. Wiernik.

Investigation: Alexander J. Denison.

Project Administration: Alexander J. Denison.

Supervision: Brenton M. Wiernik.

Writing - Original Draft Preparation: Alexander J. Denison.

Writing - Review \& Editing: Alexander J. Denison and Brenton M. Wiernik. 


\section{References}

Baer, R. A., Ballenger, J., Berry, D. T. R., \& Wetter, M. W. (1997). Detection of random responding on the MMPI--A. Journal of Personality Assessment, 68(1), 139-151. https://doi.org/10.1207/s15327752jpa6801_11

Berry, D. T. R., Wetter, M. W., Baer, R. A., Larsen, L., Clark, C., \& Monroe, K. (1992). MMPI-2 random responding indices: Validation using a self-report methodology. Psychological Assessment, 4(3), 340-345. https://doi.org/10.1037/1040-3590.4.3.340

Credé, M. (2010). Random responding as a threat to the validity of effect size estimates in correlational research. Educational and Psychological Measurement, 70(4), 596-612. https://doi.org/10.1177/0013164410366686

Curran, P. G. (2016). Methods for the detection of carelessly invalid responses in survey data. Journal of Experimental Social Psychology, 66, 4-19. https://doi.org/10.1016/j.jesp.2015.07.006

Curran, P. G., \& Hauser, K. A. (2019). I'm paid biweekly, just not by leprechauns: Evaluating validbut-incorrect response rates to attention check items. Journal of Research in Personality, 82, 103849. https://doi.org/10.1016/j.jrp.2019.103849

Gibson, A. (2019). Stop what you're doing, right now! Effects of interactive messages on careless responding [Dissertation, Wright State University]. https://corescholar.libraries.wright.edu/cgi/viewcontent.cgi?article=3260\&context=etd_all

Goldammer, P., Annen, H., Stöckli, P. L., \& Jonas, K. (2020). Careless responding in questionnaire measures: Detection, impact, and remedies. The Leadership Quarterly, 101384. https://doi.org/10.1016/j.leaqua.2020.101384 
Huang, J. L., Curran, P. G., Keeney, J., Poposki, E. M., \& DeShon, R. P. (2012). Detecting and deterring insufficient effort responding to surveys. Journal of Business and Psychology, 27(1), 99-114. https://doi.org/10.1007/s10869-011-9231-8

Johnson, J. A. (2005). Ascertaining the validity of individual protocols from web-based personality inventories. Journal of Research in Personality, 39(1), 103-129. https://doi.org/10.1016/j.jrp.2004.09.009

Kuncel, N. R., Goldberg, L. R., \& Kiger, T. (2011). A plea for process in personality prevarication. Human Performance, 24(4), 373-378. https://doi.org/10.1080/08959285.2011.597476

Maul, A. (2017). Rethinking traditional methods of survey validation. Measurement: Interdisciplinary Research and Perspectives, 15(2), 51-69. https://doi.org/10.1080/15366367.2017.1348108

Meade, A. W., \& Craig, S. B. (2012). Identifying careless responses in survey data. Psychological Methods, 17(3), 437-455. https://doi.org/10.1037/a0028085

Nichols, D., Greene, R., \& Schmolck, P. (1989). Criteria for assessing inconsistent patterns of item endorsement on the MMPI: Rationale, development, and empirical trials. Journal of Clinical Psychology, 45(2), 12. https://doi.org/10.1002/1097-4679(198903)45:2<239::AIDJCLP2270450210>3.0.CO;2-1

Osborne, J. W., \& Blanchard, M. R. (2011). Random Responding from Participants is a Threat to the Validity of Social Science Research Results. Frontiers in Psychology, 1. https://doi.org/10.3389/fpsyg.2010.00220

Rhemtulla, M., Borsboom, D., \& Bork, R. van. (2017). How to measure nothing. Measurement: Interdisciplinary Research and Perspectives, 15(2), 95-97. https://doi.org/10.1080/15366367.2017.1369785 
Schmitt, N., \& Stuits, D. M. (1985). Factors defined by negatively keyed items: The result of careless respondents? Applied Psychological Measurement, 9(4), 367-373. https://doi.org/10.1177/014662168500900405

Stern, M. J., Dillman, D. A., \& Smyth, J. D. (2007). Visual design, order effects, and respondent characteristics in a self-administered survey. Survey Research Methods, 1(3), 121-138. https://doi.org/10.18148/srm/2007.v1i3.600

Viswesvaran, C., \& Ones, D. S. (1999). Meta-analyses of fakability estimates: Implications for personality measurement. Educational and Psychological Measurement, 59(2), 197-210. https://doi.org/10.1177/00131649921969802

Ward, M. K., \& Pond, S. B. (2015). Using virtual presence and survey instructions to minimize careless responding on internet-based surveys. Computers in Human Behavior, 48, 554-568. https://doi.org/10.1016/j.chb.2015.01.070 